\title{
Revisiting non-significant effects of intranasal oxytocin using equivalence tests
}

\author{
Daniel S. Quintana \\ NORMENT, KG Jebsen Centre for Psychosis Research, Institute of Clinical Medicine, \\ Oslo, Norway
}

Corresponding author:

DS Quintana

Email address: daniel.quintana@medisin.uio.no

\begin{abstract}
The effects of intranasal oxytocin on social cognition are mixed, with several nonsignificant reports casting some doubts on the efficacy of intranasal oxytocin. Nevertheless, drawing inferences from non-significant values is problematic as non-significant results can be indicative of either statistical equivalence or insensitive data. Equivalence tests can be used to assess evidence for statistical equivalence, which can consequently facilitate theory falsification. To improve the inference of non-significant NHST $p$-values, this paper reports a set of equivalence tests performed on data from a recent meta-analysis summarizing 43 intranasal oxytocin studies. Data from $26.1 \%$ of non-significant meta-analytic effects were indicative of data insensitivity, rather than statistical equivalence. Equivalence tests were also performed on a set of previously unpublished data from one laboratory, to examine whether unpublished data yields similar outcomes. Of the 34 non-significant effects, $73.5 \%$ were due to data insensitivity. Altogether, these analyses illustrate how non-significant intranasal oxytocin results may not necessarily support a null effect. Implementing equivalence tests in oxytocin studies can facilitate theory falsification, which can advance the field by directing resources to more promising avenues of research.
\end{abstract}

\section{INTRODUCTION}

The neuropeptide oxytocin is critically involved in mammalian social behavior (Donaldson and Young, 2008; Insel, 1992). This preclinical work has contributed to a burgeoning interest in the use of oxytocin administration to address social dysfunction in a range of psychiatric disorders. In spite of considerable early promise, a recent meta-analysis of intranasal oxytocin's effect on social cognition yielded varied effects, with several non-significant summary effect sizes, among a few significant effect sizes. (Leppanen et al., 2017). While these non-significant effects may be discouraging for the field, drawing inferences from non-significant null hypothesis significance test (NHST) $p$-values can be problematic.

A NHST $p$-value is used as a criterion to either reject or not reject the null hypothesis. Rejecting the null hypothesis can a useful approach to assess whether two groups vary on a given variable, however, researchers cannot make any inferences regarding the null hypothesis, no matter how large the $p$-value. Consequently, it is uncertain if 
non-significant $p$-values in the intranasal oxytocin literature are indicative of either the absence of a relevant effect, or that the data were simply too insensitive to detect an effect. A related issue is that intranasal oxytocin studies reported in the literature contain tests that are generally statistically underpowered (Walum et al., 2016). The aggregation of effect sizes via meta-analysis can improve statistical power (Cohn and Becker, 2003), but it is unknown if sufficient intranasal oxytocin studies are available to perform appropriately powered meta-analyses on the social-cognitive effects of intranasal oxytocin. Publication bias is also an intertwined issue, as unpublished nonsignificant results may bias meta-analyses that only aggregate published studies, which are more likely to be statistically significant. However, it is also unclear the extent to which unpublished non-significant studies support the absence of an effect or simply have insensitive data.

Altogether, the purpose of this study is to improve the inference of non-significant intranasal oxytocin results by applying equivalence testing, which is a means of assessing evidence for the absence of an effect, to recent meta-analytic outcomes (Leppanen et al., 2017). Equivalence testing was also applied to a set of unpublished intranasal oxytocin outcomes (Lane et al., 2016) to examine whether unpublished data provides similar results to published meta-analytic data.

\section{MATERIALS AND METHODS}

Analyses were conducted using the R statistical environment (version 3.3.2), with data and scripts to reproduce results available at https://osf.io/2h6j9. Although it is possible to assess support for a null hypothesis within a Bayesian framework (e.g., Bürkner et al., 2017), equivalence tests allow for the rejection of effects as large, or larger, than a smallest effect size of interest using a frequentist framework. Thus, the two one-sided test (TOST) procedure for equivalence testing was implemented (Hauck and Anderson, 1984; Lakens, 2017; Schuirmann, 1987), in which a significant $p$-value is indicative of statistical equivalence. A NHST $p$-value alone can only be used to provide support for a difference, whereas combining a NHST with the TOST procedure yields four possible outcomes for an effect: i) not equivalent and not different, ii) statistically equivalent and different, iii) Not equivalent and statistically different, and iv) statistically equivalent and not different (Fig. 1). The TOST procedure was performed for all primary and moderator analyses reported in Leppanen and colleagues' meta-analysis (2017). There is no clear theoretical lower boundary for oxytocin's social-cognitive effects to determine the smallest effect size of interest (SESOI) for equivalence bounds. In lieu of this, the smallest effect size that can be detected with sufficient statistical power for a given a meta-analysis was used for the equivalence test bounds (Lakens, 2017). For this analysis, $80 \%$ power was deemed to be sufficient. For the meta-analytic effects, statistical power was calculated using a formula from Valentine and colleagues (2010). Equivalence tests using reported data were performed using the TOSTER R package (version 0.2.3; Lakens, 2017). Instead of using the default one-tailed assumption for the NHST meta-analysis, two-tailed tests were used for consistency with the original analyses.

For Lane and colleagues' paper containing previously unpublished research (2016), the smallest effect sizes that could be detected with $80 \%$ statistical power (Table 1) were 


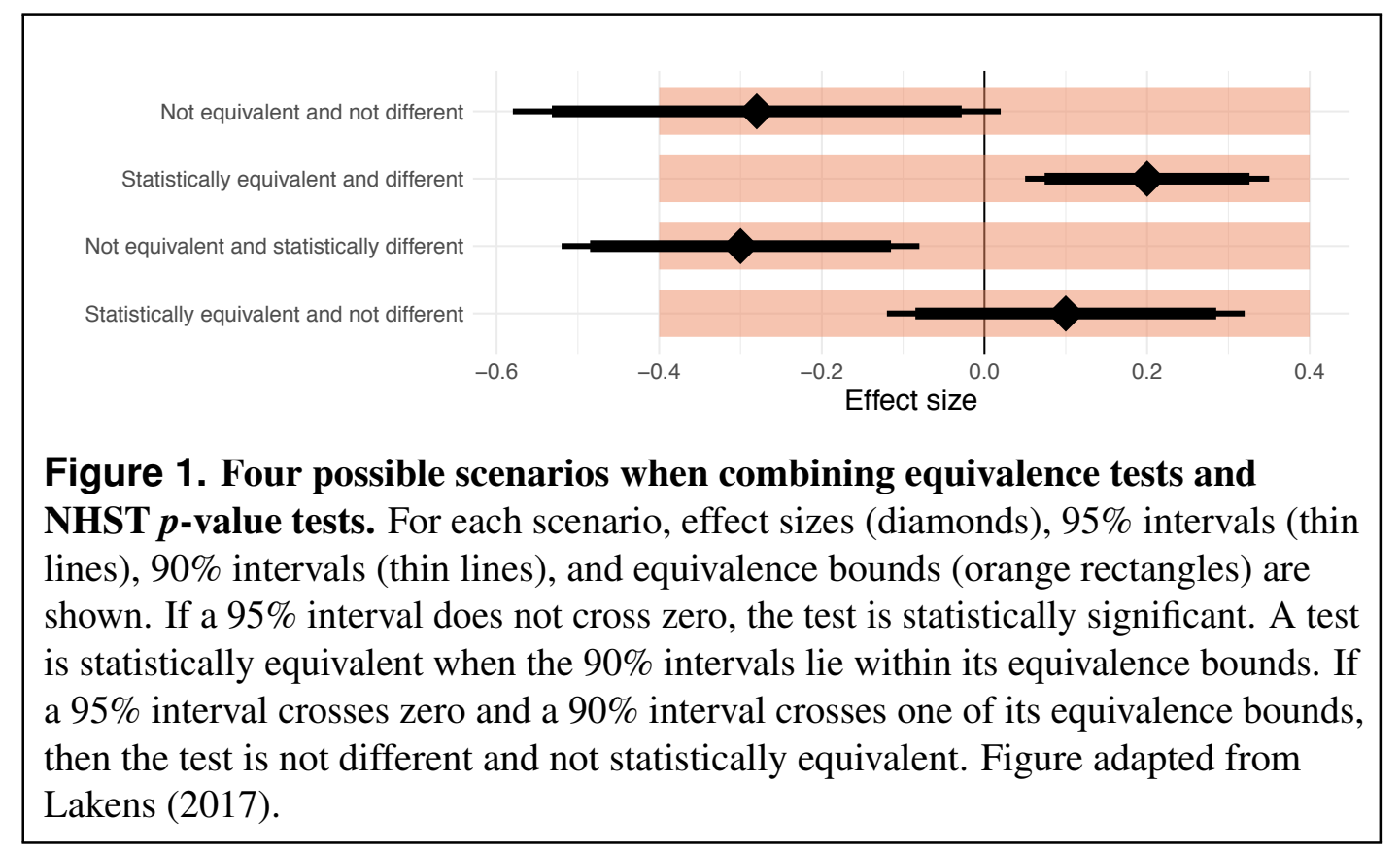

calculated using the "pwr" R package (version 1.2-1; Champely, 2016). Equivalence tests were then performed using these equivalence bounds. To control the family-wise error rate, significant alpha levels were Bonferroni corrected for the number of effect sizes reported from each study.

\section{RESULTS}

The smallest effect sizes that each meta-analysis had $80 \%$ power to detect are presented in Table 1 . On average, primary meta-analyses $(n=10)$ had $80 \%$ power to detect an effect size of at least $d=0.26$ (range: $d=0.15$ to $d=0.4$ ) and moderator meta-analyses $(\mathrm{n}=18)$ had $80 \%$ power to detect an effect size of at least $d=0.43$ (range: $d=0.17$ to $d=0.94$ ). Across all 28 meta-analytic tests (i.e., both primary and moderator analyses), 23 were non-significant (Leppanen et al., 2017). Of these 23 non-significant tests, 6 were not statistically equivalent $(26.1 \%)$, which is indicative of data insensitivity (Table 1; Fig. 2A). More specifically, eight out of ten of the primary meta-analyses were not statistically significant (Table 1; Fig. 2A). Of these eight non-significant primary meta-analyses, only the emotion sensitivity analysis was not statistically equivalent $(\mathrm{Z}=$ $0.2, p=0.42$ ) given a SESOI of $d=0.16$. Of the 18 moderator analyses, 15 were not statistically significant. Of these 15 non-significant moderator analyses, five were not statistically equivalent: the clinical $(\mathrm{Z}=0.85, p=0.2$; SESOI of $d=0.35)$ and healthy ( $\mathrm{Z}$ $=-1.31 p=0.1$; SESOI of $d=0.28$ ) subgroup analyses for theory of mind performance, emotion recognition in the clinical group ( $\mathrm{Z}=-0.47, p=0.32$; SESOI of $d=0.29)$, and the sensitivity to detect sadness $(Z=1.13, p=0.13$; SESOI of $d=0.32)$ and anger $(Z=$ $1, p=0.16$; SESOI of $d=0.32$ ). The original meta-analysis did not perform multiple test corrections for moderator analyses, which is common practice for meta-analyses in the biobehavioral sciences. Regardless, all equivalence tests were still statistically significant after Bonferroni correction of critical alpha values. 


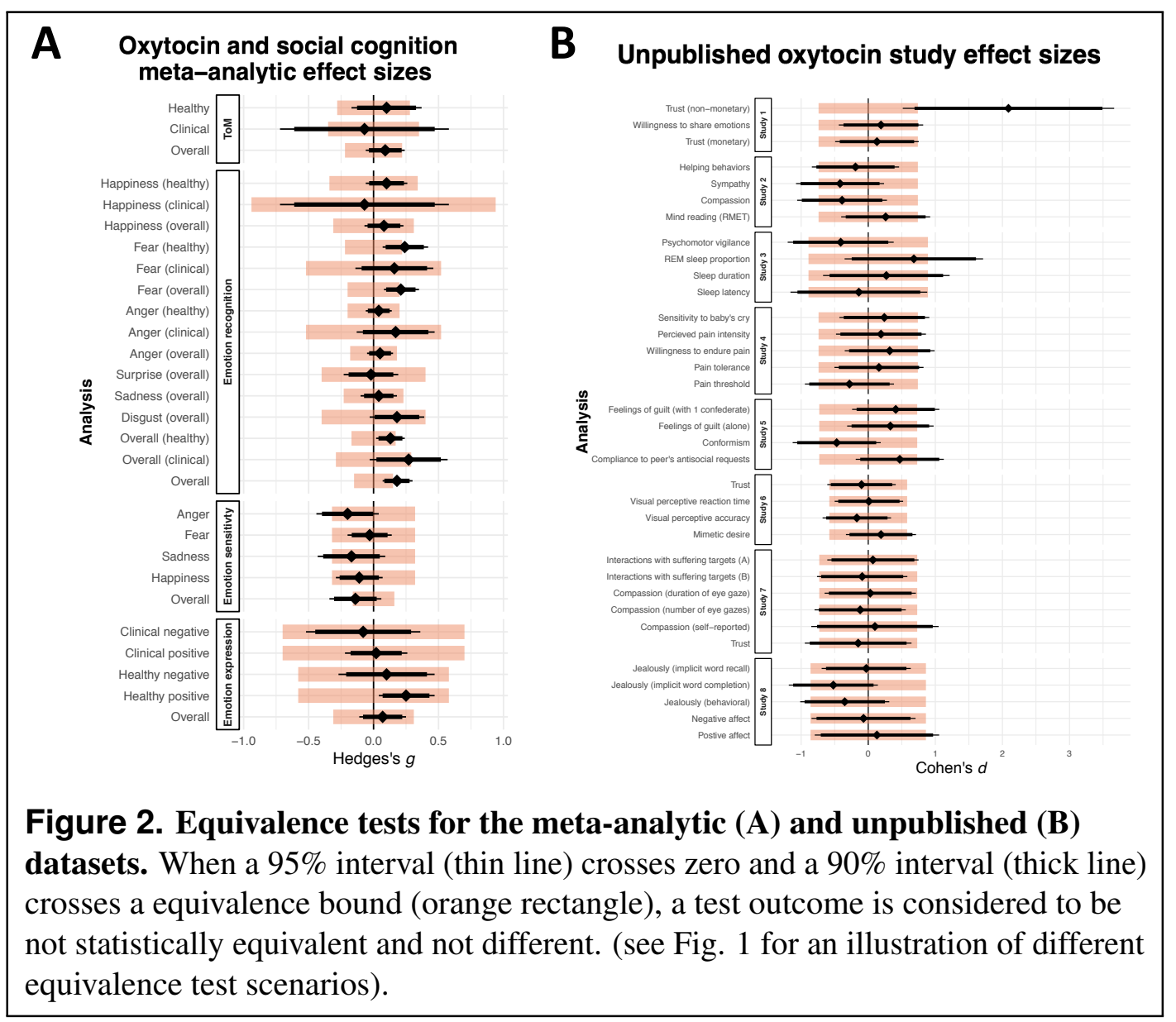

For the eight unpublished studies reported by Lane and colleagues (2016), the average smallest effect size detectable with $80 \%$ power was $d=0.78$ (range: $d=0.61$ to $d=0.94)$. Of the 34 out of 35 non-significant results, 25 of these tests $(73.5 \%)$ were not statistically equivalent (Table S1; Figure 2B).

\section{DISCUSSION}

Equivalence testing is a straightforward procedure that improves the inference of non-significant NHST $p$-values. Here, equivalence testing suggested that just over a quarter of non-significant meta-analytic findings for the interpretation and expression of emotions after intranasal oxytocin were due to data insensitivity, rather than statistical equivalence between groups. The data provided further support that the effects of oxytocin on basic emotion recognition may be limited to fear, as the recognition of anger, disgust, sadness, surprise, and happiness after intranasal oxytocin and placebo were statistically equivalent. Furthermore, while the impact of intranasal oxytocin on overall emotion sensitivity was inconclusive, its effects on emotional expression were statistically equivalent. This indicates that additional research with larger sample sizes may be required to better assess oxytocin's effects on the sensitivity to recognize basic emotions.

Of the 43 individual studies included in the original meta-analysis, only six (7.2\%) 
reported tests that had at least $80 \%$ statistical power to detect an effect size of $d=0.5$ (Leppanen et al., 2017). In contrast, all ten of the primary meta-analyses had at least $80 \%$ statistical power or more to detect a medium effect size $(d=0.5)$, demonstrating how meta-analysis can increase statistical power for the inference of both significant and non-significant effects. For the moderator meta-analyses, $61.1 \%$ had at least $80 \%$ statistical power or more to detect an effect size of $d=0.5$. While this demonstrates that underpowered moderator analyses are not necessarily widespread, researchers should be wary of whether these tests are sufficiently powered, as moderator analyses are more likely to include a smaller number of studies.

The secondary analysis of unpublished data revealed that almost three-quarters of these non-significant findings could be attributed to data insensitivity, rather than statistical equivalence. Although the release of previously unpublished results by Lane and colleagues should be applauded, these tests were statistically underpowered, a potential limitation also noted by the authors (Lane et al., 2016). On average, tests from these unpublished studies were only sufficiently powered to detect an effect of $d=0.78$, which is much larger than the average effect of intranasal oxytocin on social cognition of $d=0.28$ (Walum et al., 2016). In fact, statistical tests from none of eight reported studies were sufficiently powered to detect a medium effect size $(d=0.5)$. Regardless, several of these unpublished studies still demonstrated statistical equivalence in spite of inadequate statistical power.

The study has some limitations worth noting. First, rather than using a theoretical rationale to specify the equivalence boundaries using, they were set to the smallest effect size a given analysis had adequate power to detect. The equivalence bounds for most of the meta-analytic analyses were sufficiently sensitive to reject small-to-medium effects, which seems appropriate as this is the average effect size for oxytocin studies (Walum et al., 2016). However, the use of larger power-driven equivalence bounds for the unpublished studies instead of smaller theory-driven bounds may have led to different conclusions, with far more analyses likely to be have deemed inconclusive if smaller bounds were used. Second, the meta-analytic equivalence test results can only be generalized to the included studies in the meta-analysis, which had its own set of inclusion and exclusion criteria. There are also sure to be several tranches of unpublished intranasal oxytocin studies on a variety of areas, with the current sample not necessarily representative of unpublished intranasal oxytocin research in general.

To be clear, these mixed results should not be used absolve all non-significant oxytocin results, both past and future. Some may be tempted to exploit uncertainty surrounding non-significant results by suggesting non-significant effects could be due to data insensitivity, but as demonstrated above there are several effects that demonstrate statistically equivalency. Researchers are encouraged to use the TOSTER R package or spreadsheet calculator (Lakens, 2017) to probe specific non-significant results for themselves. Intranasal oxytocin studies have come under increasing scrutiny, particularly regarding statistical power (Walum et al., 2016). However, the focus of statistical power issues has been largely directed towards tests yielding significant results rather than non-significant results. As appropriately powered tests aid the accurate inference of non-significant results, researchers should apply the same constructive skepticism for non-significant studies as for significant studies. All that to say, issues surrounding sample size and statistical inference should not unduly detract attention from other 
methodological issues hampering intranasal oxytocin research, such as poor administration methods and the limited understanding of how intranasally administered oxytocin is transported to the brain (Quintana et al., 2016).

Equivalence tests can be used to assess evidence for reported effects, however, an additional benefit of these procedures are how they can aid theory falsification. Studies that do not specify the smallest effect size of interest are unfalsifiable (Morey and Lakens, 2016). Thus, pre-specifying equivalence bounds during the planning stages of a study can coax researchers to consider at what point an effect size would be small enough to disprove a hypothesis, along with providing a means to support a pre-specified null hypothesis (Lakens, 2017). This analysis demonstrates that the effects of intranasal oxytocin appears to be restricted to specific features of social cognition. Evidence for the absence of effects for some of these features may be disappointing for some. However, it is hoped these results can help provide the impetus to recalibrate resources from fruitless areas towards facets of social cognition that show more promise. Specific areas of social cognition that have been inconclusive to date should also be revisited with appropriately powered studies. 


\section{ACKNOWLEDGMENTS}

This work was supported by an Excellence Grant from the Novo Nordisk Foundation (NNF16OC0019856). The author would like to thanks Daniël Lakens for helpful comments on an earlier version of this manuscript. 


\section{REFERENCES}

Bürkner, P.-C., Williams, D.R., Simmons, T.C., Woolley, J.D., 2017. Intranasal Oxytocin May Improve High-Level Social Cognition in Schizophrenia, But Not Social Cognition or Neurocognition in General: A Multilevel Bayesian Meta-analysis. Schizophr. Bull.

Champely, S., 2016. pwr: Basic Functions for Power Analysis, R package version 1.2 ed.

Cohn, L.D., Becker, B.J., 2003. How meta-analysis increases statistical power. Psychol. Methods 8, 243.

Donaldson, Z.R., Young, L.J., 2008. Oxytocin, vasopressin, and the neurogenetics of sociality. Science 322, 900-904.

Hauck, W.W., Anderson, S., 1984. A new statistical procedure for testing equivalence in two-group comparative bioavailability trials. J. Pharmacokinet. Pharmacodyn. 12, 83-91.

Insel, T.R., 1992. Oxytocin - a neuropeptide for affiliation: evidence from behavioral, receptor autoradiographic, and comparative studies. Psychoneuroendocrinology 17, 3-35.

Lakens, D., 2017. Equivalence tests: A practical primer for t tests, correlations, and meta-analyses. Social Psychological and Personality Science, 1948550617697177.

Lane, A., Luminet, O., Nave, G., Mikolajczak, M., 2016. Is there a publication bias in behavioral intranasal oxytocin research on humans? Opening the file drawer of one lab. J. Neuroendocrinol. 28.

Leppanen, J., Ng, K.W., Tchanturia, K., Treasure, J., 2017. Meta-analysis of the effects of intranasal oxytocin on interpretation and expression of emotions. Neurosci. Biobehav. Rev. Morey, R.D., Lakens, D., 2016. Why most of psychology is statistically unfalsifiable. Submitted.

Quintana, D.S., Guastella, A.J., Westlye, L.T., Andreassen, O.A., 2016. The promise and pitfalls of intranasally administering psychopharmacological agents for the treatment of psychiatric disorders. Mol. Psychiatry 21, 29-38.

Schuirmann, D.J., 1987. A comparison of the two one-sided tests procedure and the power approach for assessing the equivalence of average bioavailability. J. Pharmacokinet. Pharmacodyn. 15, 657-680.

Valentine, J.C., Pigott, T.D., Rothstein, H.R., 2010. How many studies do you need? A primer on statistical power for meta-analysis. Journal of Educational and Behavioral Statistics 35, 215-247.

Walum, H., Waldman, I.D., Young, L.J., 2016. Statistical and methodological considerations for the interpretation of intranasal oxytocin studies. Biol. Psychiatry 79, 251-257. 
Table 1. Meta-analysis and equivalence tests

\begin{tabular}{|c|c|c|c|c|c|c|}
\hline & \multirow{2}{*}{ Social-cognitive domain } & \multirow{2}{*}{ SESOI } & \multicolumn{2}{|c|}{ Meta-analysis } & \multicolumn{2}{|c|}{ Equivalence test } \\
\hline & & & Z & $p$ & Z & $p$ \\
\hline \multirow{3}{*}{$\sum_{0}$} & Healthy & 0.28 & 0.73 & 0.47 & -1.31 & 0.1 \\
\hline & Clinical & 0.35 & -0.21 & 0.83 & 0.85 & 0.2 \\
\hline & Overall & 0.22 & 1.18 & 0.24 & -1.7 & 0.04 \\
\hline \multirow{15}{*}{ 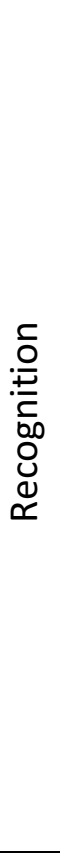 } & Happiness (healthy) & 0.34 & 1.23 & 0.22 & -2.94 & 0.002 \\
\hline & Happiness (clinical) & 0.94 & -0.21 & 0.83 & 2.64 & 0.004 \\
\hline & Happiness (overall) & 0.31 & 1.05 & 0.3 & -3.01 & 0.001 \\
\hline & Fear (healthy) & 0.22 & 2.69 & 0.007 & 0.22 & 0.59 \\
\hline & Fear (clinical) & 0.52 & 1.05 & 0.3 & -2.35 & 0.009 \\
\hline & Fear (overall) & 0.2 & 3.05 & 0.002 & 0.15 & 0.56 \\
\hline & Anger (healthy) & 0.2 & 0.78 & 0.43 & -3.1 & 0.001 \\
\hline & Anger (clinical) & 0.52 & 1.11 & 0.27 & -2.29 & 0.01 \\
\hline & Anger (overall) & 0.18 & 0.98 & 0.33 & -2.55 & 0.005 \\
\hline & Surprise (overall) & 0.4 & -0.19 & 0.85 & 3.63 & $<0.001$ \\
\hline & Sadness (overall) & 0.23 & 0.58 & 0.56 & -2.76 & 0.003 \\
\hline & Disgust (overall) & 0.4 & 1.72 & 0.09 & -2.1 & 0.02 \\
\hline & Overall (healthy) & 0.17 & 2.32 & 0.02 & -0.71 & 0.24 \\
\hline & Overall (clinical) & 0.29 & 1.79 & 0.07 & -0.13 & 0.45 \\
\hline & Overall & 0.15 & 3.07 & 0.002 & 0.51 & 0.7 \\
\hline \multirow{5}{*}{ 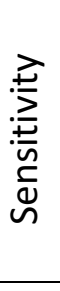 } & Anger & 0.32 & -1.67 & 0.1 & 1 & 0.16 \\
\hline & Fear & 0.32 & -0.36 & 0.72 & 3.44 & $<0.001$ \\
\hline & Sadness & 0.32 & -1.28 & 0.2 & 1.13 & 0.13 \\
\hline & Happiness & 0.32 & -1.2 & 0.23 & 2.29 & 0.01 \\
\hline & Overall & 0.16 & -1.41 & 0.16 & 0.2 & 0.42 \\
\hline \multirow{5}{*}{ 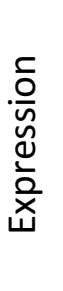 } & Clinical negative & 0.7 & -0.36 & 0.72 & 2.76 & 0.003 \\
\hline & Clinical positive & 0.7 & 0.17 & 0.87 & -5.67 & $<0.001$ \\
\hline & Healthy negative & 0.58 & 0.53 & 0.6 & -2.54 & 0.005 \\
\hline & Healthy positive & 0.58 & 2.28 & 0.02 & -3.01 & 0.001 \\
\hline & Overall & 0.31 & 0.76 & 0.45 & -2.61 & 0.004 \\
\hline
\end{tabular}

Note: $\mathrm{ToM}=$ Theory of mind, $\mathrm{SESOI}=$ Smallest effect size of interest 


\begin{tabular}{|c|c|c|c|c|}
\hline & Analysis & $\begin{array}{c}\text { Smallest detectable } \\
\text { effect size ( } 80 \% \\
\text { power) }\end{array}$ & NHST & Equivalence test \\
\hline \multirow{3}{*}{ 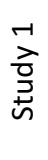 } & Trust (monetary) & \multirow{3}{*}{0.74} & $Z=0.5, p=0.62$ & $Z=-2.34, p=0.01$ \\
\hline & Willingness to share emotions & & $Z=0.72, p=0.47$ & $Z=-2.09, p=0.018$ \\
\hline & Trust (non-monetary) & & $Z=3.18, p=0.001$ & $Z=2.05, p=0.98$ \\
\hline \multirow{4}{*}{$\frac{N}{2}$} & Mind reading (RMET) & \multirow{4}{*}{0.74} & $Z=0.98, p=0.33$ & $Z=-1.81, p=0.04$ \\
\hline & Compassion & & $Z=-1.46, p=0.15$ & $Z=1.31, p=0.1$ \\
\hline & Sympathy & & $Z=-1.6, p=0.11$ & $Z=1.22, p=0.11$ \\
\hline & Helping behaviors & & $Z=-0.73, p=0.47$ & $Z=2.11, p=0.017$ \\
\hline \multirow{4}{*}{$\sum_{\substack{n \\
\frac{0}{n}}}^{m}$} & Sleep latency & \multirow{4}{*}{0.89} & $Z=-0.34, p=0.73$ & $Z=1.84, p=0.033$ \\
\hline & Sleep duration & & $Z=0.72, p=0.47$ & $Z=-1.64, p=0.05$ \\
\hline & REM sleep proportion & & $Z=1.65, p=0.1$ & $Z=-0.51, p=0.31$ \\
\hline & Psychomotor vigilance & & $Z=-1.3, p=0.19$ & $Z=1.52, p=0.06$ \\
\hline \multirow{5}{*}{ 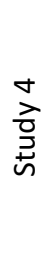 } & Pain threshold & \multirow{5}{*}{0.74} & $Z=-1.09, p=0.28$ & $Z=1.79, p=0.037$ \\
\hline & Pain tolerance & & $Z=0.62, p=0.53$ & $Z=-2.25, p=0.012$ \\
\hline & Willingness to endure pain & & $Z=1.23, p=0.22$ & $Z=-1.61, p=0.053$ \\
\hline & Perceived pain intensity & & $Z=0.73, p=0.47$ & $Z=-2.11, p=0.017$ \\
\hline & Sensitivity to baby's cry & & $Z=0.92, p=0.34$ & $Z=-1.92, p=0.027$ \\
\hline \multirow{4}{*}{$\sum_{\substack{n \\
n}}^{n}$} & Compliance to antisocial requests & \multirow{4}{*}{0.73} & $Z=1.79, p=0.07$ & $Z=-1, p=0.16$ \\
\hline & Conformism & & $Z=-1.79, p=0.07$ & $Z=1, p=0.16$ \\
\hline & Feelings of guilt (alone) & & $Z=1.28, p=0.2$ & $Z=-1.55, p=0.06$ \\
\hline & Feelings of guilt (with 1 confederate) & & $Z=1.58, p=0.12$ & $Z=-1.23, p=1.09$ \\
\hline \multirow{4}{*}{$\stackrel{0}{\frac{0}{0}}$} & Mimetic desire & \multirow{4}{*}{0.58} & $Z=0.91, p=0.36$ & $Z=-1.86, p=0.031$ \\
\hline & Visual perceptive accuracy & & $Z=-0.83, p=0.4$ & $Z=2.01, p=0.022$ \\
\hline & Visual perceptive reaction time & & $Z=0.05, p=0.96$ & $Z=-2.97, p=0.003$ \\
\hline & Trust & & $Z=-0.49, p=0.62$ & $Z=2.35, p=0.009$ \\
\hline \multirow{6}{*}{ 文 } & Compassion (self-reported) & \multirow{6}{*}{0.73} & $Z=0.39, p=0.7$ & $Z=-2.47, p=0.007$ \\
\hline & Trust & & $Z=-0.58, p=0.56$ & $Z=2.25, p=0.012$ \\
\hline & Compassion (number of eye gazes) & & $Z=-0.47, p=0.64$ & $Z=2.37, p=0.01$ \\
\hline & Compassion (duration of eye gaze) & & $Z=0.12, p=0.91$ & $Z=-2.72, p=0.003$ \\
\hline & Interactions with suffering targets & & $Z=0.27, p=0.79$ & $Z=-2.56, p=0.005$ \\
\hline & Interactions with suffering targets & & $Z=-0.35 p=0.72$ & $Z=2.51 p=0.006$ \\
\hline \multirow{5}{*}{ 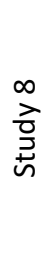 } & Positive affect & \multirow{5}{*}{0.86} & $Z=0.36, p=0.72$ & $Z=-2.03, p=0.02$ \\
\hline & Negative affect & & $Z=-0.23, p=0.82$ & $Z=2.62, p=0.004$ \\
\hline & Jealously (behavioral) & & $Z=-1.15, p=0.25$ & $Z=1.68, p=0.046$ \\
\hline & Jealously (implicit word completion) & & $Z=-1.7, p=0.1$ & $Z=1.11, p=0.13$ \\
\hline & Jealously (implicit word recall) & & $Z=-0.1, p=0.92$ & $Z=2.76, p=0.0029$ \\
\hline
\end{tabular}

